\title{
Construção e Avaliação para I 5 Listas de Palavras Baseadas no Paradigma Deese-Roediger-McDermott
}

\author{
Patricia Hong ${ }^{1, *}$ (D), Maria Alice de Mattos Pimenta Parente ${ }^{1}$, Peter Maurice Erna Claessens ${ }^{1}$ (D), \\ $\&$ Ruth Ferreira Galduróz ${ }^{1,2}$ (D) \\ ${ }^{1}$ Universidade Federal do ABC (UFABC), Santo André, SP, Brasil \\ ${ }^{2}$ Universidade Estadual Paulista (UNESP), São Paulo, SP, Brasil
}

\begin{abstract}
RESUMO - Com base nos estudos conduzidos de acordo com o paradigma Deese-Roediger-McDermott (DRM), em quais palavras de uma lista tendem a evocar outros itens relacionados, utilizado em pesquisas de falsas memórias, neste estudo elaborou-se 15 listas de palavras semanticamente relacionadas para o português. Para a elaboração das listas, foram considerados critérios como frequência de palavras no corpus de português brasileiro e extensão de palavras. A pré-seleção guiada por características linguísticas visava controlar possíveis vieses por parte dos voluntários. Testes de associação semântica e concretude de palavras foram realizados para apurar a conveniência dos itens selecionados, e um teste de recordação livre foi conduzido para avaliar características de evocação. Sugerimos a utilização das listas de palavras em pesquisas que avaliem falsas memórias.
\end{abstract}

PALAVRAS-CHAVE: falsas memórias, palavras relacionadas, paradigma Deese-Roediger-McDermott

\section{Construction and Evaluation for I5-Word Lists Based on the Deese-Roediger-McDermott Paradigm}

\begin{abstract}
Based on studies conducted in accordance with the Deese-Roediger-McDermott (DRM) paradigm, in which words of a list tend to evoke other related items, used in false memory research, this study produced 15 lists of semantically related words for Brazilian Portuguese. For the preparation of the lists, certain criteria were considered, such as word length and frequency of words in a Brazilian Portuguese corpus. Pre-selection guided by linguistic characteristics aimed to control possible biases among volunteers. Semantic association and concreteness tests were carried out to determine the suitability of the selected items, and a free recall test was performed to evaluate evocation characteristics. We suggest using word lists in researches that evaluate false memories.
\end{abstract}

KEYWORDS: false memories, related words, Deese-Roediger-McDermott paradigm

Pesquisas relacionadas à memória frequentemente utilizam listas de palavras para testar a memória explícita sob variadas situações (Almeida Valverde Zanini et al., 2012; Fernandes, Wammes, Priselac, \& Moscovitch, 2016; Luccia, Bueno, \& Santos, 2005). Estudos que investigam a ocorrência de falsas memórias, incidente natural nos seres humanos e que leva indivíduos a criarem uma memória sobre um fato ou evento que não chegou a ocorrer (Jou \& Flores, 2013; Loftus, 1997; Loftus \& Pickrell, 1995; Wade, Garry, Read, \& Lindsay, 2002), comumente examinam esse fenômeno através da apresentação de listas de palavras semanticamente relacionadas.

Proposto inicialmente nos estudos de Deese (1959a, $1959 b)$, os resultados obtidos através deste método demonstraram que, durante uma tarefa de recordação de palavras, itens de uma determinada lista provocavam a evocação de outros itens não apresentados, uma ocorrência que é chamada de intrusão. Essa tendência estaria ligada ao grau de associação da palavra crítica com as palavras nas listas apresentadas. Assim, se as palavras de uma lista

\footnotetext{
*E-mail: pateh87@gmail.com

- Submetido: 03/02/2017; Revisado: 08/05/2018; Aceito: 28/05/2018.
} 
como, por exemplo, dormir, cama e cansado se relacionam diretamente com a palavra sono, sucessivamente, a palavra crítica sono tende a ser recordada livremente, mesmo que ela não estivesse na lista apresentada ao participante do estudo.

Mais tarde, Roediger e McDermott (1995) adaptaram o procedimento para criar falsas memórias em laboratório. Este método de avaliação, conhecido como Deese-RoedigerMcDermott Paradigm, ou paradigma DRM, tem como propósito observar a recordação e o reconhecimento de palavras conhecidas como iscas críticas (lures): itens fortemente relacionados com as 15 palavras de cada uma das listas apresentadas, mas que não foram estudadas pelos indivíduos durante o teste. O paradigma DRM, utilizado como uma opção para estudar os processos de intrusão na memória, tem sido amplamente utilizado na área da psicologia cognitiva e da neurociência. Através desses dados reproduzidos em laboratório, busca-se observar em quais situações falácias na memória são mais comuns e como esse conceito se aplica sob diferentes estados comportamentais (Baioui, Ambach, Walter, \& Vaitl, 2012; Diekelmann, Landolt, Lahl, Born, \& Wagner, 2008; Marini, Agosta, Mazzoni, Barba, \& Sartori, 2012; Smeets, Otgaar, Candel, $\&$ Wolf, 2008).

Um dos métodos utilizados para a criação de listas de palavras relacionadas para a população brasileira sucedeu-se através da tradução e adaptação do artigo original em inglês, e através da associação livre, na qual listas de palavras relacionadas foram construídas a partir da resposta fornecida pelos voluntários da pesquisa (Stein, Feix, \& Rohenkohl, 2006; Stein \& Pergher, 2001). O método de associação livre é frequentemente realizado em estudos de normas de palavras. Neste método, solicita-se que os participantes forneçam de uma a três palavras recordadas que lhes vierem à memória, dada uma específica palavra-alvo (Nelson, McEvoy, \& Dennis, 2000; Salles et al., 2008). No entanto, a livre associação como um procedimento robusto para buscar palavras associadas parece limitar-se às primeiras respostas, visto que quando os sujeitos eram convidados a sugerir uma segunda palavra-resposta, a confiabilidade dos dados reduziu em relação à primeira resposta (Nelson et al., 2000). Estatisticamente, esse tipo de viés, gerado pelo encadeamento de resposta e inibição de recuperação (Marques, 2002; McEvoy \& Nelson, 1982; Nelson et al., 2000), poderia ser reduzido através de um tamanho amostral suficientemente grande ( $\approx 1000$ ), já que a livre associação não é um método padrão utilizado para identificar muitas palavras associadas de um conjunto, sobretudo em amostras pequenas (Jenkins \& Palermo, 1965; Nelson et al., 2000).

Um dos fatores importantes a serem considerados durante a elaboração de listas de palavras direciona-se à extensão das mesmas. Já está bem documentado que os processos de retenção verbal de curto prazo dependem da alça fonológica, e que esse armazenamento fonológico passivo é suscetível ao decaimento com o passar do tempo (Baddeley, 1992). Palavras demasiadamente extensas podem exigir mais da memória de trabalho e, como o efeito da extensão da palavra se concentra em fatores temporais (Baddeley \& Andrade, 1994; Baddeley, Thomson, \& Buchanan, 1975), quanto mais extenso o conteúdo, mais facilmente o indivíduo pode cair no esquecimento (Grivol \& Hage, 2011). Essa questão pode gerar outra barreira ao utilizar o método de associação livre como uma forma de obter diversas palavras associadas de um alvo, ao restringir e limitar o controle da extensão das palavras associadas em relação à palavra-alvo, uma vez que as respostas são dadas pelos voluntários da pesquisa.

Outros fatores, também essenciais, que devem ser levados em consideração durante a construção de listas de palavras para estudos sobre memória e processamento de palavras, se concentram no controle da frequência (Brysbaert $\&$ New, 2009) e no nível de concretude das palavras (Calais, Lima-Gregio, Arantes, Gil, \& Lopes Borges, 2012). Palavras com frequência alta são processadas com maior eficiência do que palavras com frequência baixa (Campoy, 2008; Cowan et al., 1992; Parente \& Salles, 2007) e palavras mais concretas apresentam um tempo de processamento e precisão peculiar quando comparado com palavras mais abstratas (Calais et al., 2012; Janczura, Castilho, Rocha, van Erven, \& Huang, 2007). Portanto, controlar essas variáveis pode proporcionar maior confiabilidade aos resultados dos estudos.

Considerando os pontos levantados acima e diante do interesse em pesquisas sobre falsas memórias, o objetivo principal deste estudo foi adaptar listas de palavras semanticamente e contextualmente relacionadas para o português brasileiro, que apresentassem controle de extensão e frequência de palavras, bem como obter medidas de concretude e associação semântica de palavras, e características de evocação falsa para jovens e adultos com escolaridade superior da região de São Paulo. Na primeira etapa do estudo, conduziu-se um método para a elaboração das listas com prévio contole para extensão e frequência de palavras, fundamentado nas ocorrências do Banco de Português do corpus brasileiro da PUC (Sardinha, 2003). Medidas de associação semâtica e concretude das palavras também foram coletadas através de questionários fechados, reduzindo as respostas geradas estritamente por significado pessoal. Na segunda etapa do estudo, realizou-se um teste de recordação livre a fim de observar as características das listas e parâmetros de recordação. Desta forma, as listas de palavras do presente estudo acrescentam uma nova ferramenta psicolinguística para a comunidade científica, sobretudo para pesquisas relacionadas com falsas memórias. 


\section{MÉTODO}

Inicialmente, a partir de 18 palavras usuais pertencentes ao vocabulário do português brasileiro (cheiro, sono, rápido, livro, guerra, país, doce, alto, música, inseto, água, copo, mão, filho, doença, camisa, claro e hora), traduzidas e adaptadas das listas de Roediger, Watson, McDermott, \& Gallo (2001) e coletadas a partir das normas de Janczura et al. (2007) e Calais et al. (2012), foram elaboradas 18 listas de palavras semanticamente e contextualmente relacionadas (Sardinha, 2003). Essas 18 palavras usuais foram classificadas como palavras-alvo, porquanto a partir de cada uma delas selecionou-se uma variedade de possíveis palavras associadas, através dos dicionários da língua portuguesa bem como dicionários de sinônimos e antônimos. O período de consulta das palavras-alvo e possíveis palavras associadas ocorreu entre fevereiro de 2015 a junho de 2015.

Frequências: Com a finalidade de evitar o uso de palavras desconhecidas ou pouco utilizadas pela população, todas as palavras foram observadas quanto ao seu número de ocorrências através da busca por formas no Banco de Português, projeto DIRECT (Development of International Research for English, Commerce and Technology) - corpus brasileiro da PUC (Sardinha, 2003). A busca pelas palavras no corpus ocorreu no período de março de 2015 a outubro de 2015.

O número de ocorrências de todas as palavras foi convertido no logaritmo de frequências a fim de possibilitar a análise estatística (Roediger et al., 2001). Palavras com frequências abaixo de $60 \%$ em relação à palavra com maior ocorrência (631.277 ocorrências/ano) foram excluídas.

Extensão da palavra: Todas as palavras selecionadas para o estudo (palavras-alvo e suas palavras associadas) não ultrapassaram o máximo de três sílabas; além disso, palavras derivadas de uma mesma raiz como, por exemplo, pressa e depressa, pertencentes de uma mesma lista, foram eliminadas, adotando-se apenas uma das variações.

Possíveis palavras associadas: Em seguida, computou-se o total de palavras associadas obtidas para cada palavraalvo. A lista com maior número de palavras associadas foi da palavra-alvo Sono com 22 palavras relacionadas, e a lista com menor número de palavras associadas foi da palavra-alvo Cheiro com 16 palavras relacionadas. Isto ocorreu porque a palavra associada colonia da lista Cheiro apresentou relação entre-listas com a palavra-alvo País, reduzindo assim ainda mais a possibilidade de palavras associadas. Visto que a frequência da palavra-alvo Cheiro foi menor do que a palavra-alvo Pais e considerando o critério de 17 possíveis palavras remanescentes, optamos por eliminar do estudo a lista Cheiro, em vista das próximas etapas e visando maior confiabilidade das palavras intralistas e menor relação entre-listas. A média de palavras associadas para cada palavra-alvo foi de 17,72 $(\mathrm{DP}=1,52)$.

Após levar em consideração os critérios acima, permaneceram 17 listas de palavras, nas quais, cada lista continha um conjunto de 17 possíveis palavras associadas para cada palavra-alvo (Sono, Rápido, Livro, Guerra,
País, Doce, Alto, Música, Inseto, Água, Copo, Mão, Filho, Doença, Camisa, Claro e Hora). Essas 17 listas de palavrasalvo foram reduzidas para um total de 15 listas de palavras, conforme os critérios de exclusão adotados nas próximas etapas, e as 17 possíveis palavras associadas para cada lista foram reduzidas em 15 palavras associadas, baseado no critério de Roediger \& McDermott (1995). A Tabela 1 apresenta as 15 listas de palavras elaboradas neste estudo.

Considerando a extensão das palavras, selecionaramse apenas trissilábicos (3 a 9 letras); 169 palavras foram consideradas curtas (com até seis letras) e 71 palavras foram consideradas longas (mais de sete letras; ver Salles et al., 2008). A classe gramatical das palavras variou de acordo com a definição estabelecida pelos dicionários da língua portuguesa: 207 substantivos, 11 adjetivos, 9 verbos, 2 advérbios e 11 palavras classificadas como substantivos e adjetivos.

\section{I.Teste de Associação Semântica e Concretude de Palavras}

Com o intuito de testar o julgamento da população de São Paulo acerca das palavras, realizamos dois testes para examinar se as listas estavam adequadas para uso: Teste de associação semântica de palavras e Teste de concretude de palavras.

Participantes. A amostra foi composta por 32 voluntários de ambos os sexos (11 homens e 22 mulheres), residentes na região metropolitana de São Paulo. A idade dos voluntários variou entre 18 e 59 anos $(\mathrm{M}=28,40, \mathrm{DP}=$ 11,01). A amostra foi selecionada por conveniência, e todos os voluntários proveram seu consentimento em participar do estudo. A escolaridade dos voluntários (acima de 12 anos, com ensino superior completo ou cursando) foi adotada como critério de inclusão do estudo. A não conclusão dos respectivos testes foi adotado como critério de exclusão do estudo. O protocolo foi aprovado pelo Comitê de Ética em Pesquisa envolvendo Seres Humanos da UFABC (CEP/ CONEP CAAE 32043114.3.0000.5594, 11/09/2014).

Procedimento. Os participantes foram avaliados individualmente através de uma ferramenta de pesquisa online (SurveyMonkey Inc) ou com questionários impressos em papel, através de seu julgamento quanto ao grau de associação semântica das 17 palavras com determinadas palavras-alvo, bem como de concretude das palavras. Não foram observadas diferenças quanto ao método de avaliação adotado (online $\mathrm{n}=22$ e papel $\mathrm{n}=10: t(30)=-0,28 ; p=0,77$ ).

Teste de associação semântica de palavras. Assim como previamente fundamentada a importância da associação semântica em listas de palavras relacionadas, buscou-se estudar medidas psicolinguísticas para investigação direta do campo lexical dos indivíduos (Janczura, 2005; Nelson, Schreiber, \& McEvoy, 1992). O teste de associação semântica de palavras foi empregado através de uma escala Likert de sete pontos (1- altamente não associada, 2- não associada, 
Tabela 1

Listas de palavras

\begin{tabular}{|c|c|c|c|c|c|c|c|c|c|c|c|c|c|c|c|}
\hline \multirow{2}{*}{ Posição } & \multicolumn{15}{|c|}{ Listas } \\
\hline & 1 & 2 & 3 & 4 & 5 & 6 & 7 & 8 & 9 & 10 & 11 & 12 & 13 & 14 & 15 \\
\hline Isca & País & Sono & Hora & Livro & Filho & Doce & Doença & Guerra & Música & Claro & Camisa & Alto & Água & Mão & Inseto \\
\hline 1 & nação & dormir & minuto & título & pai & açúcar & saúde & tratado & som & escuro & calça & baixo & banho & palma & bicho \\
\hline 2 & mundo & sonho & data & leitura & família & paladar & tumor & conflito & ritmo & brilhante & terno & acima & sede & tato & antenas \\
\hline 3 & região & despertar & futuro & página & geração & geleia & enfermo & luta & maestro & lâmpada & roupa & subir & rio & garras & traça \\
\hline 4 & pátria & cama & mês & conto & bebê & sorvete & dor & combate & piano & trevas & malha & ápice & gota & punho & teia \\
\hline 5 & estado & folga & prazo & edição & irmão & cana & gripe & tropas & refrão & farol & saia & torre & molhado & anel & animal \\
\hline 6 & local & acordar & tempo & resumo & criança & mel & médico & vitória & concerto & luz & blusa & céu & vapor & pés & picada \\
\hline 7 & povo & preguiça & século & caderno & neto & salgado & vírus & disputa & tom & nítido & casaco & colina & saliva & unhas & mosca \\
\hline 8 & cidade & repouso & semana & escritor & cria & torta & remédio & batalha & canção & acender & paletó & suspenso & lago & braço & formiga \\
\hline 9 & governo & insônia & segundo & texto & parente & bolo & febre & arma & nota & sombra & bolso & gigante & chuva & polegar & aranha \\
\hline 10 & província & fadiga & duração & história & mãe & glicose & cura & militar & banda & limpo & tecido & topo & enchente & toque & barata \\
\hline 11 & mapa & deitar & relógio & autor & herdeiro & gostoso & sintoma & duelo & timbre & vela & colete & grande & mar & patas & mosquito \\
\hline 12 & terra & sossego & ano & fábula & fruto & néctar & vacina & soldado & orquestra & raio & algodão & elevar & úmido & manejo & larva \\
\hline 13 & distrito & relaxar & instante & folha & prole & sabor & infecção & derrota & violão & sol & gravata & pico & líquido & dedos & asas \\
\hline 14 & migração & cansado & hoje & narração & avós & amargo & tosse & confronto & ópera & lanterna & linho & tamanho & fluido & pulso & cupins \\
\hline 15 & colônia & coberta & momento & romance & jovem & calda & câncer & rivais & áudio & lua & traje & montanha & hídrico & pegar & lagarta \\
\hline
\end{tabular}


3- parcialmente não associada, 4- mediana, 5- parcialmente associada, 6- associada e 7- altamente associada).

Todas as palavras ou listas que apresentaram grau de associação semântica abaixo da mediana (4 pontos na escala Likert) foram eliminadas como um critério de exclusão a fim de evitar palavras com baixo relacionamento semântico e contextual. Além disso, para se obter as 15 palavras associadas por lista, as duas palavras que apresentassem menor grau de associação semântica com a palavra-alvo de cada lista foram excluídas. Os resultados do teste de associação demonstraram que as listas das palavras-alvo $\operatorname{Copo}\left(\mathrm{M}_{\text {assoc. }}=3,03 ; 3,38 ; 3,62 ; 3,86\right)$ e Rápido $\left(\mathrm{M}_{\text {assoc. }}=2,76\right.$; $3,00 ; 3,38 ; 3,48)$ apresentaram algumas palavras associadas com julgamentos abaixo da mediana (parcialmente não associada). Sendo assim, estas duas listas foram eliminadas do estudo para evitar palavras com pouca relação.

Teste de concretude de palavras. Visto que o processo de memorização pode passar por uma representação verbal ou baseada em imagem e que o desempenho da memória varia de acordo com o nível de concretude (Fliessbach, Weis, Klaver, Elger, \& Weber, 2006; Jessen et al., 2000), o teste buscou obter medidas de concretude para o controle de estudos que tenham interesse nesses parâmetros. O teste de concretude de palavras foi empregado utilizando uma escala Likert de sete pontos (1- altamente abstrata, 2- abstrata, 3parcialmente abstrata, 4- mediana, 5- parcialmente concreta, 6- concreta e 7- altamente concreta). Nesta etapa, tanto as palavras-alvo (iscas críticas) quanto as palavras associadas foram ponderadas quanto ao grau de concretude.

\section{Teste de Recordação Livre das Listas de Palavras}

Por fim, para testar as listas elaboradas pelo presente estudo, e observar o padrão de recordação serial de cada lista, realizamos o teste de recordação livre das 15 listas remanescentes.
Participantes. Foram selecionados 26 voluntários de ambos os sexos (11 homens e 15 mulheres), com ensino superior (completo ou cursando) e residentes na região metropolitana de São Paulo. A idade dos voluntários variou entre 18 e 40 anos $(M=25,20 ; \mathrm{DP}=7,45)$. Todos os voluntários assinaram o Termo de Consentimento Livre e Esclarescido para participar do estudo. Critérios de inclusão: a) escolaridade acima de 12 anos (com ensino superior completo ou cursando); b) ausência ou correção de alterações perceptuais (uso de óculos). Critérios de exclusão: a) não conclusão do teste de memória; b) resultados ilegíveis.

Procedimento. Todas as listas de palavras associadas foram apresentadas aos voluntários de forma aleatória e individual através de um monitor. As 15 palavras de cada lista foram apresentadas no centro da tela, uma em seguida da outra, com duração de dois segundos cada palavra. A fim de observar o padrão de recordação falso gerado pelas listas de palavras, as palavras-alvo (iscas críticas) de cada lista não foram apresentadas aos voluntários nesse momento.

Ao final de cada lista, os sujeitos foram instruídos sobre um clique (estímulo sonoro) sinalizando o momento de recordação das palavras previamente apresentadas, anotando em uma folha de papel o máximo de itens que conseguissem recordar. Foi dado um tempo de dois minutos para recordar cada uma das listas.

Procedimentos de análise de dados. A conversão da frequência de palavras em logaritmo de frequências deu-se através da fórmula " $\log (0,5+$ frequência bruta)", descritas por Roediger et al. (2001), a fim de minimizar a distância na distribuição de ocorrência das palavras. Posteriormente, análises de variância foram conduzidas para observar as diferenças entre médias de log-frequências e a extensão de palavras entre as listas, com o intuito de observar a equivalência das listas. Além disso, a partir dos resultados obtidos nos testes de associação de palavras e concretude de palavras, calculou-se a média das respostas fornecidas pelos voluntários, a fim de obter as medidas de associação e concretude das palavras.

\section{RESULTADOS}

Uma análise de variância (ANOVA) unifatorial foi conduzida para avaliar a hipótese nula de que não há diferença entre as listas na variável dependente logfrequência de palavras $(\mathrm{M}=4,12, \mathrm{DP}=0,68, n=225)$. A ANOVA foi significativa para $\log$-frequência de palavras, com $F(14,210)=11,63, p<0,001, \eta^{2}=0,43$, apresentando tamanho de efeito grande baseado no guia de interpretação de Cohen para valores correspondentes de eta-squared (1988). As variáveis apresentam igualdade de variâncias conforme o teste de Levene (log-frequência de palavras $=1,25, p=0,23$; extensão de palavras $=1,64, p=0,69$ ) e distribuição normal para log-frequência de palavras $(\mathrm{W}=0,94, p=0,42)$, mas a hipótese-nula de normalidade foi rejeitada para a variável extensão de palavras (ShapiroWilk $p<0,001)$. Para testar as diferenças entre as listas na variável extensão de palavras $(\mathrm{M}=5,69, \mathrm{DP}=1,39, \mathrm{n}=225)$ prosseguiu-se uma análise através do teste não-paramétrico Kruskal-Wallis, e o resultado mostrou haver significância somente marginal para extensão de palavras, $\mathrm{x}^{2}(14)=$ $23,42, p=0,054$. A Figura 1 mostra a média de extensão e $\log$-frequência para cada lista de palavras.

Visto que o resultado da análise para extensão de palavras apresentou significância marginal, uma segunda análise foi conduzida para esta variável $(\mathrm{N}=210)$ sem a lista Mão, que tinha uma média do número de letras discrepante em relação às outras listas $(\mathrm{M}=4,88, \mathrm{DP}=1,41)$. Os dados 

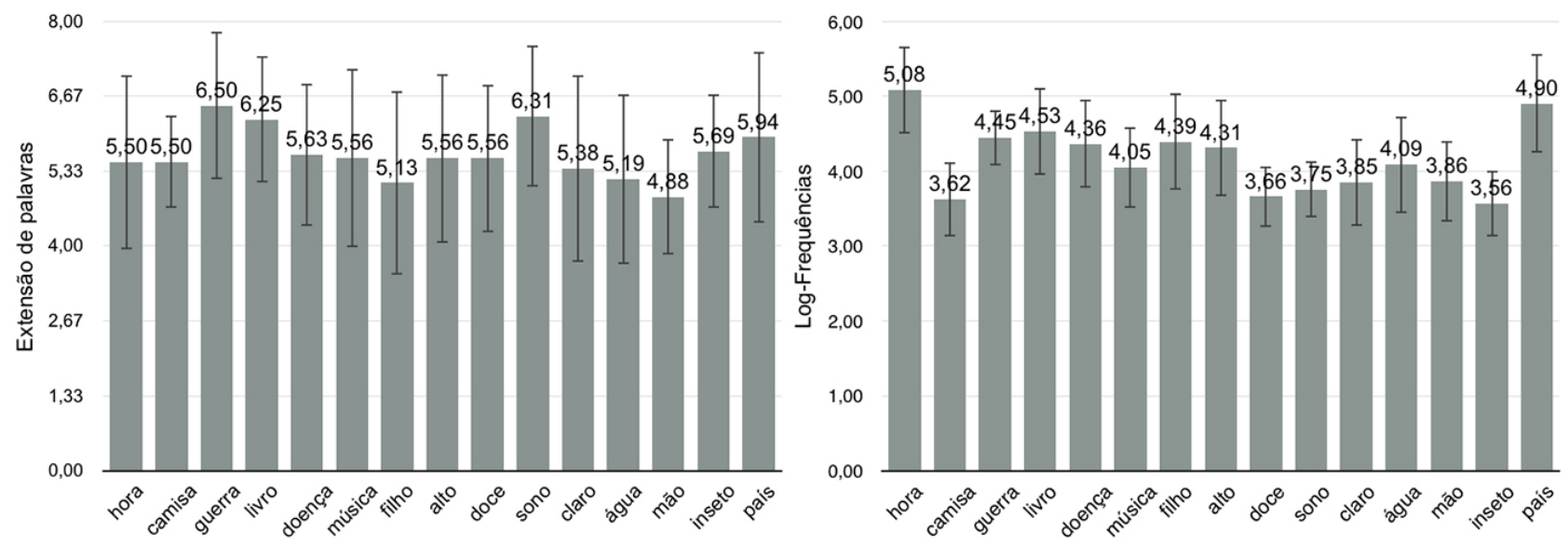

Figura 1. Média e barra de erro (desvio padrão) de extensão de palavras (painel esquerdo) e de log-frequência (painel direito) por lista.

apresentaram homocedasticidade $(\mathrm{W}=1,14, p=0,32)$ porém distribuição não normal ( $\mathrm{W}=0,94, p<0,001)$, conduzindose então uma análise não paramétrica. Desta vez, o resultado do teste Kruskal-Wallis não mostrou haver diferença significativa entre as listas $-\mathrm{x}^{2}(13)=18,29, \mathrm{p}=0,14$.

A média de associação semântica entre a palavra-alvo com suas respectivas 15 palavras associadas foi de 5,83 (DP $=0,75)$. A palavra com menor taxa de associação semântica entre a palavra-alvo e seu associado foi a palavra amargo (assoc. 4,00 $\pm 2,16$ ) da lista Doce. A palavra com maior taxa de associação alvo-associado foi a palavra dormir (assoc. $6,90 \pm 0,30)$ da lista Sono. A média de associação obtida entre a palavra-alvo com as suas 15 palavras associadas é demonstrada através da Tabela 2.

Em relação aos resultados do teste de concretude, a faixa de pontuação das palavras estudadas variou de 2,25 a $6,75(\mathrm{M}=4,98, \mathrm{DP}=1,23)$, sendo que a palavra com maior taxa de abstração foi tempo $(\mathrm{M}=2,25, \mathrm{DP}=1,46)$ da lista Hora e as palavras com maior taxa de concretude foram calça, terno e gravata (todas $\mathrm{M}=6,75$ ) da lista Camisa. Os escores obtidos pela escala-Likert apresentaram distribuição de frequência bimodal. Seguindo os critérios de corte estabelecidos na escala Likert (entre 1 a $3=$ abstrata; $4=$ média; entre 5 a 7 = concreta) e com base nos julgamentos obtidos, $26,70 \%$ das palavras foram consideras abstratas,
$47,90 \%$ das palavras foram consideradas concretas e $25,40 \%$ das palavras não foram consideradas nem abstratas nem concretas. As médias de concretude das palavras são apresentadas na Tabela 3 .

Considerando os resultados do teste de recordação livre, a pontuação média de recordação das palavras estudadas pelos 26 sujeitos foi de 7,61 (DP $=1,5)$. As palavras críticas que apresentaram maior número de falsa recordação foram Sono e Alto, ambas com 12 intrusões. A palavra crítica que apresentou menor taxa de falsa recordação foi Mão com duas intrusões. A média de intrusões obtidas a partir das listas foi de $6,80(\mathrm{DP}=3,41)$. Os resultados da proporção de recordação são apresentados na Tabela 4. Além disso, as listas que apresentaram maior quantidade de distratores relacionados, palavras associadas semanticamente, mas não apresentadas (distintas de palavras críticas), foram Sono (com 15 palavras) e Hora (com 14 palavras), e a lista que apresentou menor taxa de distratores relacionados foi Filho, com 6 instrusões. Não houve presença de distratores nãorelacionados, respostas que afeririam "chute" ou desatenção por parte dos voluntários. O padrão obtido com a soma das respostas de recordação (em forma de U) é demonstrada na Figura 2. Entre a primeira e a terceira palavra inicial, observa-se um declínio de recordação, que se preserva horizontalmente, e retoma curva ascendente a partir da

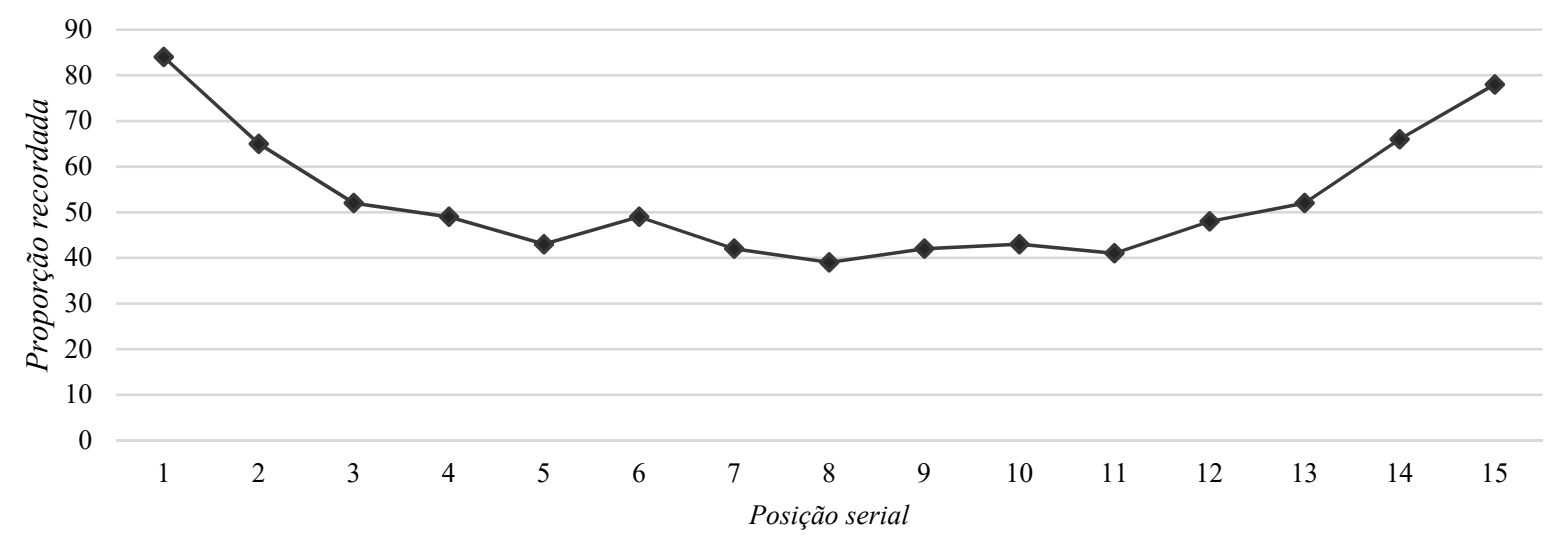

Figura 2. Curva de recordação em função da posição serial das palavras. 
Tabela 2

A. Média e desvio padrão da pontuação obtida no teste de associação de palavras

\begin{tabular}{|c|c|c|c|c|c|c|c|c|c|c|c|c|c|c|c|}
\hline Posição & País & Sono & Hora & Livro & Filho & Doce & Doença & Guerra & Música & Claro & Camisa & Alto & Água & Mão & Inseto \\
\hline 1 & $6,63(0,71)$ & $6,9(0,3)$ & $6,73(0,51)$ & $6,37(0,8)$ & $6,83(0,45)$ & $6,83(0,45)$ & $6,07(1,49)$ & $5,13(1,24)$ & $6,67(0,47)$ & $5,7(2,06)$ & $5,7(1,46)$ & $5,67(1,88)$ & $6,37(0,61)$ & $6,67(0,47)$ & $6,23(0,84)$ \\
\hline 2 & $5,31(1,55)$ & $6,43(0,87)$ & $5,6(1,22)$ & $6,63(0,48)$ & $6,43(0,71)$ & $6,17(0,74)$ & $6,43(0,71)$ & $6,6(0,56)$ & $6,73(0,44)$ & $5,9(1,25)$ & $6,2(0,93)$ & $5,97(1,36)$ & $6,63(0,55)$ & $6,57(0,5)$ & $6,2(0,97)$ \\
\hline 3 & $6,03(0,93)$ & $6,4(0,98)$ & $4,67(1,57)$ & $6,63(0,55)$ & $6,07(1,05)$ & $5,97(1,18)$ & $6,67(0,54)$ & $6,5(0,62)$ & $6,47(0,67)$ & $6,2(0,72)$ & $6,67(0,64)$ & $6,03(1,19)$ & $6,6(0,49)$ & $5,47(1,46)$ & $6,3(0,82)$ \\
\hline 4 & $6,59(0,67)$ & $6,4(0,87)$ & $4,83(1,31)$ & $5,9(0,93)$ & $6,13(1,31)$ & $6,33(0,7)$ & $6,43(0,83)$ & $6,67(0,62)$ & $6,37(0,79)$ & $4,4(2,14)$ & $5,57(1,52)$ & $6,27(0,89)$ & $6,4(0,76)$ & $6,4(1)$ & $5,7(1,31)$ \\
\hline 5 & $5,91(1,12)$ & $4,27(1,37)$ & $5,2(1,26)$ & $6,23(0,86)$ & $5,63(1,46)$ & $6,23(0,78)$ & $6,47(0,72)$ & $6,43(0,61)$ & $6,57(0,57)$ & $5,83(1)$ & $4,33(1,98)$ & $5,93(0,98)$ & $6,53(0,5)$ & $5,93(1,05)$ & $5,77(1,55)$ \\
\hline 6 & $5,47(1,16)$ & $6,17(1,22)$ & $6,53(0,62)$ & $5,2(1,42)$ & $5,83(1,49)$ & $6,53(0,56)$ & $6,27(0,9)$ & $6,1(1,18)$ & $6,37(0,7)$ & $6,5(0,84)$ & $5,87(1,25)$ & $6,13(0,91)$ & $6,1(0,82)$ & $5,28(1,93)$ & $6,5(0,72)$ \\
\hline 7 & $5,81(1,12)$ & $5,23(1,27)$ & $4,67(1,27)$ & $4,8(1,53)$ & $5,47(1,7)$ & $4,07(2,29)$ & $6,3(0,84)$ & $6,4(0,67)$ & $6,5(0,67)$ & $6,1(1)$ & $5,23(1,41)$ & $6,03(0,84)$ & $5,57(1,22)$ & $6,13(0,83)$ & $6,53(0,62)$ \\
\hline 8 & $5,34(1,6)$ & $5,93(0,96)$ & $4,9(1,35)$ & $6,53(0,8)$ & $5,6(1,78)$ & $5,4(1,64)$ & $6,4(0,83)$ & $6,57(0,67)$ & $6,63(0,55)$ & $5,93(0,95)$ & $5,73(1,28)$ & $5,17(1,62)$ & $6,57(0,5)$ & $5,8(1,26)$ & $6,57(0,84)$ \\
\hline 9 & $5,84(1,19)$ & $6,07(1,27)$ & $6,53(0,72)$ & $6,23(0,62)$ & $5,6(1,21)$ & $6,1(1)$ & $6,3(0,84)$ & $6,53(0,62)$ & $6,5(0,67)$ & $4,67(1,9)$ & $5,43(1,56)$ & $6,1(1,22)$ & $6,67(0,47)$ & $6,37(0,8)$ & $5,87(1,59)$ \\
\hline 10 & $5,06(1,86)$ & $4,97(1,22)$ & $6,13(1)$ & $6,23(0,76)$ & $6,7(0,58)$ & $6,4(0,67)$ & $5,83(1,47)$ & $6,5(0,57)$ & $6,4(0,55)$ & $4,5(1,81)$ & $6(1,03)$ & $6,33(0,78)$ & $6,43(0,76)$ & $6,13(0,97)$ & $6,5(0,72)$ \\
\hline 11 & $5,38(1,6)$ & $5,47(1,39)$ & $6,8(0,47)$ & $6,67(0,54)$ & $6,4(0,84)$ & $5,9(1,23)$ & $6,33(0,83)$ & $5,87(1,36)$ & $6,43(0,67)$ & $5(1,36)$ & $5,33(1,31)$ & $6,07(1,22)$ & $6,6(0,55)$ & $5(1,58)$ & $6,57(0,61)$ \\
\hline 12 & $5,16(1,51)$ & $4,37(1,29)$ & $4,73(1,53)$ & $5,6(1,1)$ & $5,3(1,57)$ & $5,2(1,69)$ & $5,77(1,42)$ & $6,57(0,5)$ & $6,4(0,61)$ & $5,27(1,3)$ & $5,7(1,2)$ & $6(1,55)$ & $6,13(0,87)$ & $5,33(1,77)$ & $6(0,93)$ \\
\hline 13 & $4,97(1,51)$ & $5,27(1,26)$ & $5,1(1,11)$ & $5,93(1,18)$ & $5,7(1,45)$ & $6,1(0,91)$ & $6,13(0,83)$ & $5,9(1,04)$ & $6,4(0,71)$ & $6,2(0,97)$ & $5,7(1,45)$ & $6,07(1,01)$ & $6,33(0,75)$ & $6,6(0,49)$ & $6,03(1,18)$ \\
\hline 14 & $4,97(1,66)$ & $5,6(1,34)$ & $5,1(1,32)$ & $5,7(1,04)$ & $5,23(1,56)$ & $4(2,16)$ & $6(1,05)$ & $6,5(0,57)$ & $6,3(0,69)$ & $5,67(1,06)$ & $5,5(1,3)$ & $5,83(1)$ & $5,93(1)$ & $6,2(0,71)$ & $6,3(0,97)$ \\
\hline 15 & $4,94(1,46)$ & $5,3(1,38)$ & $5,27(1,11)$ & $5,97(1,14)$ & $4,8(1,88)$ & $5,47(1,57)$ & $6,37(0,9)$ & $6,17(0,82)$ & $6,33(0,7)$ & $5,27(1,38)$ & $5,77(1,28)$ & $6(0,92)$ & $6,53(0,62)$ & $6,4(0,67)$ & $6(1,03)$ \\
\hline Média & $5,61(0,56)$ & $5,68(0,77)$ & $5,54(0,8)$ & $6,05(0,54)$ & $5,92(0,57)$ & $5,8(0,83)$ & $6,24(0,25)$ & $6,31(0,41)$ & $6,48(0,13)$ & $5,56(0,66)$ & $5,64(0,51)$ & $5,97(0,27)$ & $6,35(0,31)$ & $5,99(0,53)$ & $6,22(0,3)$ \\
\hline
\end{tabular}

B. Palavras correspondentes aos valores de associação

\begin{tabular}{|c|c|c|c|c|c|c|c|c|c|c|c|c|c|c|c|}
\hline Posição & País & Sono & Hora & Livro & Filho & Doce & Doença & Guerra & Música & Claro & Camisa & Alto & Água & Mão & Inseto \\
\hline 1 & nação & dormir & minuto & título & pai & açúcar & saúde & tratado & som & escuro & calça & baixo & banho & palma & bicho \\
\hline 2 & mundo & sonho & data & leitura & família & paladar & tumor & conflito & ritmo & brilhante & terno & acima & sede & tato & antenas \\
\hline 3 & região & despertar & futuro & página & geração & geleia & enfermo & luta & maestro & lâmpada & roupa & subir & rio & garras & traça \\
\hline 4 & pátria & cama & mês & conto & bebê & sorvete & dor & combate & piano & trevas & malha & ápice & gota & punho & teia \\
\hline 5 & estado & folga & prazo & edição & irmão & cana & gripe & tropas & refrão & farol & saia & torre & molhado & anel & animal \\
\hline 6 & local & acordar & tempo & resumo & criança & mel & médico & vitória & concerto & luz & blusa & céu & vapor & pés & picada \\
\hline 7 & povo & preguiça & século & caderno & neto & salgado & vírus & disputa & tom & nítido & casaco & colina & saliva & unhas & mosca \\
\hline 8 & cidade & repouso & semana & escritor & cria & torta & remédio & batalha & canção & acender & paletó & suspenso & lago & braço & formiga \\
\hline 9 & governo & insônia & segundo & texto & parente & bolo & febre & arma & nota & sombra & bolso & gigante & chuva & polegar & aranha \\
\hline 10 & província & fadiga & duração & história & mãe & glicose & cura & militar & banda & limpo & tecido & topo & enchente & toque & barata \\
\hline 11 & mapa & deitar & relógio & autor & herdeiro & gostoso & sintoma & duelo & timbre & vela & colete & grande & mar & patas & mosquito \\
\hline 12 & terra & sossego & ano & fábula & fruto & néctar & vacina & soldado & orquestra & raio & algodão & elevar & úmido & manejo & larva \\
\hline 13 & distrito & relaxar & instante & folha & prole & sabor & infecção & derrota & violão & sol & gravata & pico & líquido & dedos & asas \\
\hline 14 & migração & cansado & hoje & narração & avós & amargo & tosse & confronto & ópera & lanterna & linho & tamanho & fluido & pulso & cupins \\
\hline 15 & colônia & coberta & momento & romance & jovem & calda & câncer & rivais & áudio & lua & traje & montanha & hídrico & pegar & lagarta \\
\hline
\end{tabular}


Tabela 3

Média e desvio padrão da pontuação obtida no teste de concretude de palavras

\begin{tabular}{|c|c|c|c|c|c|c|c|c|c|c|c|c|c|c|c|}
\hline Posição & País & Sono & Hora & Livro & Filho & Doce & Doença & Guerra & Música & Claro & Camisa & Alto & Água & Mão & Inseto \\
\hline isca & $5,28(1,35)$ & $91(1,93)$ & $25(2,09)$ & $75(0,43)$ & $6(1,03)$ & 91 & 56( & 4,31 & ,8) & 7) & 63) & 8) & 2) & $3(0,7)$ & $6,09(1,01)$ \\
\hline 1 & $5(1,46)$ & $5,06(1,64)$ & $4,44(2$ & $4,13(1,63)$ & 1) & 25) & & & & 63) & 43) & ,58) & 31) & & \\
\hline 2 & $6(1,69)$ & $2,94(1,87)$ & $4,47(1,8)$ & $3,47(1,58)$ & $47(1,87)$ & $3,44(1,6)$ & $5,59(1,48)$ & $3,91(1,31)$ & & $3,94(1,52)$ & $6,75(0,43)$ & $3,88(1,67)$ & $3,84(1,72)$ & $3,78(1,56)$ & $6,09(1,13)$ \\
\hline 3 & $4,22(1,9)$ & $4(1,63)$ & $2,28(1,37)$ & $5,69(1,4)$ & $3,5(1,73)$ & $6,25(1,15)$ & $4,44(1,56)$ & $4,72(1,3)$ & $6,09(1,16)$ & $6,59(0,55)$ & $5,47(1,44)$ & $4,13(1,69)$ & $6,34(0,73)$ & $5,78(1,27)$ & $6,16(1,23)$ \\
\hline 4 & $3,41(1,52)$ & $6,53(1,25)$ & $4,53(1,85)$ & $3,13(1,22)$ & $6,09(0,98)$ & $6,53(0,66)$ & $3,44(1,77)$ & $4,69(1,26)$ & 6,41 & $3,63(1,54)$ &, $5(1,46)$ & $4,53(1,58)$ & $5,94(1,25)$ & 85) & $6,19(1,01)$ \\
\hline 5 & $4,25(1,7)$ & $3,5(1,79)$ & $3,59(1,69)$ & $3,78(1,47)$ & $5,81(1,24)$ & $6,25(1,3)$ & $4,88(1,62)$ & $5,63(1,17)$ & $3,78(1,76)$ & $6,06(1,06)$ & $6,72(0,51)$ & $6,13(0,96)$ & $4,53(1,6)$ & $6,72(0,51)$ & $5,53(1,56)$ \\
\hline 6 & $4,41(2)$ & $4,63(1,67)$ & $2,25(1,46)$ & $4,44(1,52)$ & $5,75(1,32)$ & $6,41(0,82)$ & $6,34(0,73)$ & $3,09(1,31)$ & $4,53(1,58)$ & $4,25(1,77)$ & $6,47(0,87)$ & $5,34(1,41)$ & $5,31(1,47)$ & $5,66(1,65)$ & $4,88(1,62)$ \\
\hline 7 & $4,5(1,54)$ & $3,25(1,8)$ & $4,31(1,84)$ & $6,5(0,79)$ & $5,75(1,3)$ & $4,25(1,68)$ & $5,44(1,58)$ & $3,94(1,66)$ & 3,19 (1 & $3,63(1,56)$ & $6,59(0,7)$ & $5,88(1,22)$ & $5,91(1,13)$ & $9(0,53)$ & $0,61)$ \\
\hline 8 & $5,22($ & 3,88 & 4,5 & & 4,47 & & & 6) & & 6) & 48) & & 73) & & \\
\hline 9 & $4,16(1,58)$ & $3,41(1,66)$ & $4,19(1,99)$ & $4,97(1,53)$ & $4,47(1,71)$ & $6,44(0,83)$ & $5(1,39)$ & $6,13(1,02)$ & $3,84(1,6)$ & $4,34(1,49)$ & $6,28(1,01)$ & $84(1,6)$ & $6,13(0,89)$ & $6,56(0,75)$ & $6,63(0,54)$ \\
\hline 10 & $4,19(1,53)$ & $3,47(1,79)$ & $3,44(1,37)$ & $4,19(1,4)$ & $5,94(1,2)$ & $5,34(1,65)$ & $3,44(1,82)$ & $4,81(1,51)$ & $5,97(1,07)$ & $3,91(1,59)$ & $5,59(1,56)$ & $5,25(1,5)$ & $5,56(1,47)$ & $4,16(1,77)$ & $6,63(0,54)$ \\
\hline 11 & $6(1,44)$ & $5,41(1,41)$ & $6,66(0,64)$ & $5,88(1,22)$ & $4,56(1,66)$ & $3,03(1,59)$ & $3,94(1,68)$ & $4,47(1,62)$ & $3,5(1,52)$ & $6,38(0,78)$ & $6,63(0,6)$ & $4,44(1,68)$ & $6,38(0,86)$ & $6,38(0,89)$ & $6,5(0,66)$ \\
\hline 12 & $5,72(1,57)$ & $2,84(1,44)$ & $4,63(1,83)$ & $3,97(1,55)$ & $4,69(1,83)$ & $5,06(1,8)$ & $5,88(1,14)$ & $6,09(0,95)$ & $6,19(0,85)$ & $5,88(1,19)$ & $5,84(1,56)$ & $4,5(0,95)$ & $4,25(1,64)$ & $3,28(1,68)$ & $6,09(1,35)$ \\
\hline 13 & $4,34(1,61)$ & $3,31(1,59)$ & $2,53(1)$ & $6(1,12)$ & $4,59(1,75)$ & $3,16(1,56)$ & $4,44(1,78)$ & $3,09(1,31)$ & $6,63(0,54)$ & $6,06(1,12)$ & $6,75(0,43)$ & $5,03(1,42)$ & $5,16(1,58)$ & $6,63(0,65)$ & $6,19(1,18)$ \\
\hline 14 & $4,03(1,81)$ & $3,69(1,7)$ & $4(1,85)$ & $3,97(1,63)$ & $5,84(1,18)$ & $3,53(1,58)$ & $5,28(1,33)$ & $4,06(1,69)$ & $5,13(1,29)$ & $6,56(0,7)$ & $5,41(1,56)$ & $3,38(1,49)$ & $4,03(1,81)$ & $6,13(1,36)$ & $6,53(0,66)$ \\
\hline 15 & $4,06(1,62)$ & $5,84(1,18)$ & $2,47(1,35)$ & $3,44(1,17)$ & $3,16(1,44)$ & $5,5(1,56)$ & $5,34(1,53)$ & $4(1,6)$ & $3,94(1,71)$ & $6(1,27)$ & $5,06(1,6)$ & $6,41(0,74)$ & $4,19(1,84)$ & $4(1,75)$ & $6,53(0,71)$ \\
\hline Média & $4,54(0,70)$ & $3,99(1,12)$ & $4,01(1,18)$ & $4,87(1,12)$ & $5,2(0,95)$ & $5,08(1,35)$ & $4,71(0,95)$ & $4,52(0,93)$ & $4,77(1,20)$ & $4,86(1,21)$ & $6,27(0,61)$ & $4,63(0,89)$ & $5,43(0,91)$ & $5,84(1,21)$ & $6,11(0,57)$ \\
\hline
\end{tabular}

B. Palavras correspondentes aos valores de concretude

\begin{tabular}{|c|c|c|c|c|c|c|c|c|c|c|c|c|c|c|c|}
\hline & País & Sono & Hora & Livro & Filho & Doce & Doença & Guerra & Música & Claro & Camisa & Alto & Água & Mão & Inseto \\
\hline 1 & nação & dormir & minuto & título & pai & açúcar & saúde & tratado & som & escuro & calça & baixo & banho & palma & bicho \\
\hline 2 & mundo & sonho & data & leitura & família & paladar & tumor & conflito & ritmo & brilhante & terno & acima & sede & tato & antenas \\
\hline 3 & região & despertar & futuro & página & geração & geleia & enfermo & luta & maestro & lâmpada & roupa & subir & rio & garras & traça \\
\hline 4 & pátria & cama & mês & conto & bebê & sorvete & dor & combate & piano & trevas & malha & ápice & gota & punho & teia \\
\hline 5 & estado & folga & prazo & edição & irmão & cana & gripe & tropas & refrão & farol & saia & torre & molhado & anel & animal \\
\hline 6 & local & acordar & tempo & resumo & criança & mel & médico & vitória & concerto & luz & blusa & céu & vapor & pés & picada \\
\hline 7 & povo & preguiça & século & caderno & neto & salgado & vírus & disputa & tom & nítido & casaco & colina & saliva & unhas & mosca \\
\hline 8 & cidade & repouso & semana & escritor & cria & torta & remédio & batalha & canção & acender & paletó & suspenso & lago & braço & formiga \\
\hline 9 & governo & insônia & segundo & texto & parente & bolo & febre & arma & nota & sombra & bolso & gigante & chuva & polegar & aranha \\
\hline 10 & província & fadiga & duração & história & mãe & glicose & cura & militar & banda & limpo & tecido & topo & enchente & toque & barata \\
\hline 11 & mapa & deitar & relógio & autor & herdeiro & gostoso & sintoma & duelo & timbre & vela & colete & grande & mar & patas & mosquito \\
\hline 12 & terra & sossego & ano & fábula & fruto & néctar & vacina & soldado & orquestra & raio & algodão & elevar & úmido & manejo & larva \\
\hline 13 & distrito & relaxar & instante & folha & prole & sabor & infecção & derrota & violão & sol & gravata & pico & líquido & dedos & asas \\
\hline 14 & migração & cansado & hoje & narração & avós & amargo & tosse & confronto & ópera & lanterna & linho & tamanho & fluido & pulso & cupins \\
\hline 15 & colônia & coberta & momento & romance & jovem & calda & câncer & rivais & áudio & lua & traje & montanha & hídrico & pegar & lagarta \\
\hline
\end{tabular}


$11^{a}$ palavra. Esta disposição em recordar mais palavras apresentadas no começo e no final das listas demonstra integridade dos efeitos de primazia e recência que serão discutidos mais adiante. As médias de recordação falsa para três listas (Alto, Guerra e Doce) foram semelhantes às médias de recordação de itens apresentados no meio da lista.

Tabela 4

Proporção de itens recordados, em porcentagem, em função da posição serial

\begin{tabular}{|c|c|c|c|c|c|c|c|c|c|c|c|c|c|c|c|c|}
\hline Posição & País & Sono & Hora & Camisa & Alto & Água & Doença & Filho & Música & Guerra & Inseto & Mão & Doce & Livro & Claro & Média \\
\hline Isca & 19 & 35 & 27 & 27 & 46 & 8 & 35 & 27 & 15 & 42 & 15 & 8 & 46 & 31 & 12 & 26,2 \\
\hline 1 & 100 & 92 & 92 & 88 & 81 & 62 & 54 & 96 & 85 & 85 & 100 & 50 & 92 & 85 & 92 & 83,60 \\
\hline 2 & 77 & 81 & 58 & 85 & 50 & 38 & 77 & 69 & 50 & 81 & 96 & 77 & 42 & 42 & 58 & 65,40 \\
\hline 3 & 54 & 42 & 50 & 38 & 35 & 65 & 50 & 58 & 54 & 50 & 62 & 42 & 62 & 65 & 46 & 51,53 \\
\hline 4 & 42 & 31 & 42 & 65 & 42 & 46 & 65 & 38 & 50 & 19 & 50 & 62 & 23 & 81 & 73 & 48,60 \\
\hline 5 & 77 & 12 & 31 & 58 & 46 & 50 & 65 & 38 & 46 & 38 & 42 & 38 & 38 & 23 & 35 & 42,47 \\
\hline 6 & 65 & 46 & 62 & 42 & 42 & 38 & 46 & 50 & 42 & 46 & 54 & 31 & 54 & 54 & 62 & 48,93 \\
\hline 7 & 35 & 54 & 38 & 27 & 42 & 46 & 38 & 54 & 42 & 27 & 50 & 42 & 62 & 31 & 42 & 42,00 \\
\hline 8 & 65 & 54 & 35 & 42 & 19 & 35 & 31 & 62 & 31 & 23 & 38 & 50 & 38 & 31 & 38 & 39,47 \\
\hline 9 & 38 & 23 & 27 & 46 & 19 & 65 & 42 & 50 & 23 & 54 & 81 & 50 & 58 & 31 & 15 & 41,47 \\
\hline 10 & 50 & 54 & 38 & 31 & 23 & 62 & 38 & 65 & 42 & 58 & 62 & 15 & 50 & 27 & 23 & 42,53 \\
\hline 11 & 31 & 31 & 42 & 19 & 31 & 42 & 46 & 77 & 50 & 31 & 81 & 46 & 31 & 19 & 35 & 40,80 \\
\hline 12 & 46 & 23 & 50 & 50 & 42 & 81 & 31 & 46 & 73 & 42 & 46 & 46 & 50 & 54 & 38 & 47,87 \\
\hline 13 & 23 & 31 & 73 & 62 & 77 & 50 & 58 & 58 & 73 & 27 & 38 & 65 & 42 & 46 & 54 & 51,80 \\
\hline 14 & 42 & 65 & 46 & 88 & 62 & 85 & 62 & 92 & 73 & 58 & 42 & 54 & 77 & 81 & 65 & 66,13 \\
\hline 15 & 58 & 69 & 73 & 81 & 81 & 85 & 100 & 69 & 77 & 77 & 42 & 85 & 96 & 92 & 92 & 78,47 \\
\hline Média & 51,38 & 46,44 & 49,00 & 53,06 & 46,13 & 53,63 & 52,38 & 59,31 & 51,63 & 47,38 & 56,19 & 47,56 & 53,81 & 49,56 & 48,75 & 51,08 \\
\hline
\end{tabular}

\section{DISCUSSÃO}

Os resultados obtidos neste estudo mostraram que o prévio controle de padrões específicos e importantes se torna válido e necessário para a construção de listas de palavras semanticamente e contextualmente relacionadas que serão utilizadas em testes de falsas memórias. Embora os resultados de comparação de médias para extensão de palavras não tenham alcançado significância, observou-se uma tendência das diferenças entre extensão média das listas serem significativas, principalmente por causa da lista com a palavra-alvo Mão. Ao prosseguir as análises sem a lista Mão, essa tendência não foi mais observada. Dessa forma, para estudos em que seja necessário ter um maior controle sobre o pareamento das extensões das palavras, sugerimos a utilização do instrumento sem a lista Mão. Para estudos em que não seja necessário conhecer a extensão das listas, sugerimos a utilização do instrumento completo.

Eliminar palavras com baixo grau de associação semântica entre a palavra-alvo e palavras associadas são de suma importância, pois quanto maior a conexão entre a palavra-alvo e palavras associadas, maior a viabilidade de se observar e estudar o fenômeno de falsas memórias. A recordação de itens críticos deve aumentar conforme a força da correlação associativa dos itens relacionados com a palavra-alvo aumenta (Deese, 1959a, 1959b), e essa característica foi observada de forma particular para a palavra-alvo Sono, que apresentou maior taxa de isca crítica e maior taxa de associação com a palavra associada dormir.
No presente estudo não foi determinada a associação reversa, cálculo da força de conexão das palavras estudadas com a palavra-alvo (Roediger et al., 2001; Stein \& Gomes, 2009). No entanto, durante o processo de criação e montagens das listas, teve-se o prévio cuidado de selecionar palavras diretamente relacionadas com a palavra-alvo. Especificamente, duas listas que apresentaram palavras com baixa associação semântica durante a análise dos resultados foram eliminadas para manter a força associativa de todas as listas com suas palavras-alvo (Roediger et al., 2001). Assim, sugerimos que esses dois critérios adotados minimizam o risco de baixa associação reversa, bem com o encadeamento gerado pela resposta-sobre-a-resposta (Nelson et al., 2000). Além disso, sugerimos que o método de elaboração das listas adotado pelo presente estudo minimiza o risco do voluntário de fornecer respostas de acordo com um significado pessoal e não de um consenso social acerca dos significados frente a uma palavra geradora, visto que para diferentes voluntários uma determinada resposta pode variar de acordo com a sua percepção e experiência individual e ao fornecer previamente possíveis palavras pertencentes a uma classificação semântica de senso comum, o agrupamento dos elementos de uma categoria pode ser facilitado e menos individualizado.

Outro fator levado em consideração para a elaboração das listas foi a frequência de palavras. Palavras mais frequentes apresentam vantagens durante a recordação e palavras pouco frequentes apresentam melhor memória de reconhecimento. 
Isso implica numa maior taxa de êxito e menor taxa de reconhecimento errôneo para palavras de frequência baixa (Yonelinas, 2002). Além disso, considerando que estudos sobre falsas memórias se focam nas intrusões (Roediger \& McDermott, 1995) e observam erros de comissão (recordação incorreta) e não de omissão de informações (Jou \& Flores, 2013; Roediger \& McDermott, 2000), obter um controle das frequências de palavras em estudos sobre memória é de suma importância, visto que a frequência permeia a acessibilidade da palavra e pode interferir nos resultados obtidos de formas variadas (Brysbaert \& New, 2009; Nelson \& McEvoy, 2000).

Apesar dos resultados obtidos demonstrarem diferenças significativas nas frequências das palavras, considerando o critério de frequência mínima para compor as listas de palavras (acima de 1.000 ocorrências no corpus), somado com o critério de eliminação de palavras com ocorrência abaixo de $60 \%$ em relação à palavra com maior ocorrência, sugerimos que todos os itens apresentem familiaridade pela população local. Propomos que as diferenças nas frequências estejam relacionadas a uma variação vinculada à classe gramatical de cada palavra, uma vez que as listas continham verbos, substantivos, adjetivos e advérbios. Além disso, estas listas versam tanto sobre esferas mais e menos presentes na linguagem (ex. "hoje" versus "larva"), mas sempre com frequência acima de um limiar mínimo para evitar palavras desconhecidas na lista. Esta medida mantém a representatividade para o corpus de uma linguagem em geral, e ao mesmo tempo, minimiza possíveis interferências que possam estar encobertos pela falta de familiaridade com a palavra.

Em relação à concretude das palavras, usualmente, pessoas apresentam melhor performance para palavras concretas do que palavras abstratas em tarefas de leitura e escrita. Isso ocorre devido a uma facilidade de acesso que as pessoas apresentam quando existe um protótipo da imagem mais bem estabelecido (Carthery-Goulart \& Parente 2006; Nelson \& Schreiber, 1992; Parente \& Salles, 2007). No presente estudo, a lista da palavra-alvo Sono, que apresentou palavras associadas com menor taxa de concretude $(\mathrm{M}=$ 3,99 ), também foi a lista que obteve maior taxa de recordação falsa, tanto para iscas críticas como para distratores relacionados. Sendo assim, consideramos importante medir esses parâmetros para que os estudos possam obter um controle dos dados trabalhados. Contudo, porquanto que estudos sobre falsas memórias podem utilizar listas de palavras para testar diferentes grupos ou situações, também se torna importante manter tanto as palavras concretas quanto as palavras abstratas em situações experimentais.

Por fim, o resultado da análise do teste de recordação livre apresentou uma curva de posição serial em forma de U, indicando um efeito de primazia e recência, padrão de recordação comumente observado em estudos sobre memória com recordação livre de palavras não associadas (Atkinson \& Shiffrin, 1968; Murdock, 1962; Roediger \& McDermott, 1995; Stein et al., 2006). Sabe-se que o efeito de primazia ocorre quando as palavras no início da lista são melhor recordadas devido ao gradiente atencional e ausência de interferência (Azizian \& Polich, 2007; Page \& Norris, 1998), e o efeito de recência ocorre quando as palavras no fim da lista são melhor recordadas por estarem disponíveis na condição imediata para recuperação e por não sofrerem interferência subsequente (Azizian \& Polich, 2007; Shiffrin \& Atkinson, 1969). Além disso, assim como no estudo de Roediger e McDermott (1995), os resultados obtidos neste estudo demonstraram que em algumas listas, itens não apresentados aos sujeitos foram recordados na mesma proporção que itens apresentados de posições seriais medianas, sendo este um indicativo do poder de estímulo que leva a intrusão.

\section{CONCLUSÃO}

O resultado do presente estudo oferece 15 listas de palavras semanticamente e contextualmente relacionadas às palavras críticas bem como dados e medidas dessas listas quanto ao grau de associação semântica, concretude, frequência e extensão em adultos de alta escolaridade da região de São Paulo. A utilização dessas listas fornece uma base para estudos que buscam analisar eventos variados ocasionados pela memória, sobretudo de falsas memórias.
O método adotado para criação das listas através de critérios previamente estabelecidos do presente estudo, mostrou-se adequado aos objetivos; sendo assim, consideramos um instrumento conveniente para uso da comunidade cientifica. Para pesquisas futuras, sugerimos controlar a associação reversa e observar esses parâmetros em faixas etárias variadas (crianças e idosos), bem como diversificar o método de avaliação do paradigma DRM para listas de caráter visual.

\section{REFERÊNCIAS}

Almeida Valverde Zanini, G. de, Tufik, S., Andersen, M. L., da Silva, R. C., Bueno, O. F., Rodrigues, C. C., \& Pompéia, S. (2012). Free recall of word lists under total sleep deprivation and after recovery sleep. Sleep, 35(2), 223-230. doi: 10.5665/sleep. 1626

Atkinson, R. C., \& Shiffrin, R. M. (1968). Human memory: A proposed system and its control processes. In K. W. Spence. \&
J. T. Spence (Eds.), The psychology of learning and motivation (pp. 89 -195). New York: Academic Press.

Azizian, A., \& Polich, J. (2007). Evidence for attentional gradient in the serial position memory curve from event-related potentials. Journal of Cognitive Neuroscience, 19(12), 2071-2081. doi:10.1162/jocn.2007.19.12.2071 
Baddeley, A. D. (1992). Working memory. Science, 255(5044), 556-559.

Baddeley, A. D., \& Andrade, J. (1994). Reversing the word-length effect: A comment on Caplan, Rochon, and Waters. Quarterly Journal of Experimental Psychology, 47A, 1047-1054.

Baddeley, A. D., Thomson, N., \& Buchanan, M. (1975). Word length and the structure of short-term memory. Journal of Verbal Learning and Verbal Behavior, 14(6), 575-589.

Baioui, A., Ambach, W., Walter, B., \& Vaitl, D. (2012). Psychophysiology of false memories in a Deese-RoedigerMcDermott Paradigm with visual scenes. PLoS One, 7(1), e30416. doi:10.1371/journal.pone.0030416

Brysbaert, M., \& New, B. (2009). Moving beyond Kucera and Francis: A critical evaluation of current word frequency norms and the introduction of a new and improved word frequency measure for American English. Behavior Research Methods, 41(4), 977-990. doi:10.3758/BRM.41.4.977

Calais, L. L., Lima-Gregio, A. M., Arantes, P., Gil, D., \& Lopes Borges, A. C. L. C. (2012). Um julgamento de concretude de palavras. Jornal da Sociedade Brasileira de Fonoaudiologia, 24(3), 262-268. doi:10.1590/S2179-64912012000300012

Campoy, G. (2008). The effect of word length in short-term memory: Is rehearsal necessary? Quarterly Journal of Experimental Psychology, 61(5), 724-734. doi:10.1080/17470210701402364

Carthery-Goulart, M. T., \& Parente, M. A. M. P. (2006). Leitura e escrita e o envelhecimento. In M. A. M. P. Parente (Ed.), Cognição e envelhecimento (pp. 191-202). Porto Alegre, RS: Artmed.

Cohen, J. (1988). Statistical power analysis for the behavioral sciences. Hillsdale, NJ: Lawrence Earlbaum Associates.

Cowan, N., Day, L., Saults, J. S., Keller, T. A., Johnson, T., \& Flores, L. (1992). The role of verbal output time in the effects of word length on immediate memory. Journal of Memory and Language, 31, 1-17.

Deese, J. (1959a). On the prediction of occurrence of particular verbal intrusions in immediate recall. Journal of Experimental Psychology, 58, 17-22. doi:10.1037/h0046671

Deese, J. (1959b). Influence of inter-item associative strength upon immediate free recall. Psychological Reports, 5, 305-312. doi:10.2466/PR0.5.3.305-312

Diekelmann, S., Landolt, H-P., Lahl, O., Born, J., \& Wagner, U. (2008). Sleep loss produces false memories. PLoS One, 3(10), e3512. doi:10.1371/journal.pone.0003512

Fernandes, M. A., Wammes, J. D., Priselac, S., \& Moscovitch, M. (2016). Interfering with free recall of words: Detrimental effects of phonological competition. Neuropsychologia, 90, 59-71. doi:10.1016/j.neuropsychologia.2016.05.009

Fliessbach, K., Weis, S., Klaver, P., Elger, C. E., \& Weber, B. (2006). The effect of word concreteness on recognition memory. Neuroimage, 32(3), 1413-1421. doi:10.1016/j. neuroimage.2006.06.007

Grivol, M. A., \& Hage, S. R. V (2011). Phonological working memory: A comparative study between different age groups. Jornal da Sociedade Brasileira de Fonoaudiologia, 23(3), 245-251.

Janczura, G. A. (2005). Contexto e normas de associação para palavras: A redução do campo semântico. Paidéia, 15(32), 417-425. doi:10.1590/S0103-863X2005000300011

Janczura, G. A., Castilho, G. M., Rocha, N. O., van Erven, T. J. C., \& Huang, T. P. (2007). Normas de concretude para 909 palavras da língua portuguesa. Psicologia: Teoria e Pesquisa, 23(2), 195-204.

Jenkins, J. J., \& Palermo, D. S. (1965). Further data on changes in word-association norms. Journal of Personality and Social Psychology, 1(4), 303-309. doi:10.1037/h0021914
Jessen, F., Heun, R., Erb, M., Granath, D. O., Klose, U., Papassotiropoulos, A., \& Grodd, W. (2000). The concreteness effect: Evidence for dual coding and context availability. Brain and Language, 74(1), 103-112. doi:10.1006/brln.2000.2340

Jou, J., \& Flores, S. (2013). How are false memories distinguishable from true memories in the Deese-Roediger-McDermott paradigm? A review of the findings. Psychological Research, 77(6), 671-686. doi:10.1007/s00426-012-0472-6

Loftus, E. F. (1997). Creating false memories. Scientific American, 277(3), 70-75.

Loftus, E. F., \& Pickrell, J. E. (1995). The formation of false memories. Psychiatric Annals, 25, 720-725.

Luccia, G. C. P., Bueno, O. F. A., \& Santos, R. F. (2005). Recordação livre de palavras e memória operacional em idosos. Distúrbios da Comunicação, 17(3), 347-358.

Marini, M., Agosta, S., Mazzoni, G., Barba, G. D., \& Sartori, G. (2012). True and false DRM Memories: Differences detected with an implicit task. Frontiers in Psychology, 3, 310. doi:10.3389/fpsyg.2012.00310

Marques, J. F. (2002). Normas de associação livre para 302 palavras portuguesas. Revista Portuguesa de Psicologia, 36, 35-43.

McEvoy, C. L., \& Nelson, D. L. (1982). Category name and instance norms for 106 categories of various sizes. American Journal of Psychology, 95, 581-634.

Murdock, B. B. (1962). The serial position effect of free recall. Journal of Experimental Psychology, 64(5), 482-488.

Nelson, D. L., \& McEvoy, C. L. (2000). What is this thing called frequency? Memory \& Cognition, 28(4), 509-522. doi:10.3758/ BF03201241

Nelson, D. L., McEvoy, C. L., \& Dennis. S. (2000). What is free association and what does it measure? Memory \& Cognition, 28(6), 887-99. doi:10.3758/BF03209337

Nelson, D. L., \& Schreiber, T. A. (1992). Word concreteness and word structure as independent determinants of recall. Journal of Memory and Language, 31(2), 237-260. doi:10.1016/0749596X(92)90013-N

Nelson, D. L., Schreiber, T. A., \& McEvoy, C. L. (1992). Processing implicit and explicit representations. Psychological Review, 99(2), 322-48.

Page, M. P., \& Norris, D. (1998). The primacy model: A new model of immediate serial recall. Psychological Review, 105(4), 761781. doi:10.1037/0033-295X.105.4.761-781

Parente, M. A. M. P., \& Salles, J. F. (2007). Processamento da linguagem em tarefas de memória. In A. Oliveira (Ed.), Memória: Cognição e comportamento (pp. 231-255). São Paulo, SP: Casa do Psicólogo.

Roediger, H. L., III, \& McDermott, K. B. (1995). Creating false memories: Remembering words not presented in lists. Journal of Experimental Psychology: Learning, Memory, \& Cognition, 21(4), 803-814. doi:10.1037/0278-7393.21.4.803

Roediger, H. L., III, \& McDermott, K. B. (2000). Tricks of memory. Current Directions in Psychological Science, 9(4), 123-127.

Roediger, H. L., III, Watson, J. M., McDermott, K. B., \& Gallo, D. A. (2001). Factors that determine false recall: A multiple regression analysis. Psychonomic Bulletin \& Review, 8(3), 385-407. doi:10.3758/BF03196177

Salles, J. F., Holderbaum, C. S., Becker, N., Rodrigues, J. C., Liedtke, F. V., Zibetti, M. R., \& Piccoli, L. F. (2008). Normas de associação semântica para 88 palavras do português brasileiro. Psico, 39(3), 362-370.

Sardinha, T. B. (2003). The bank of Portuguese. Retrieved from http://lael.pucsp.br/direct.

Shiffrin, R. M., \& Atkinson, R. C. (1969). Storage and retrieval processes in long-term memory. Psychological Review, 76(2), 179-193. doi:10.1037/h0027277 
Smeets, T., Otgaar, H., Candel, I., \& Wolf, O. T. (2008). True or false? Memory is differentially affected by stress-induced cortisol elevations and sympathetic activity at consolidation and retrieval. Psychoneuroendocrinology, 33(10), 1378-1386. doi:10.1016/j.psyneuen.2008.07.009

Stein, L. M., Feix, L. F., \& Rohenkohl, G. (2006). Avanços metodológicos no estudo das falsas memórias: construção e normatização do procedimento de palavras associadas. Psicologia: Reflexão e Crítica, 19(2), 166-176.

Stein, L. M., \& Gomes, C. F. A. (2009). Normas brasileiras para listas de palavras associadas: Associação semântica, concretude, frequência e emocionalidade. Psicologia: Teoria e Pesquisa, 25(4), 537-546.
Stein, L. M., \& Pergher, G. K. (2001). Criando falsas memórias em adultos por meio de palavras associadas. Psicologia: Reflexão e Crítica, 14(2), 353-366.

SurveyMonkey Inc. (2015). SurveyMonkey. Retrieved from https:// pt.surveymonkey.com/. Palo Alto, Califórnia, EUA.

Wade, K. A., Garry, M., Read, J. D., \& Lindsay, D. S. (2002). A picture is worth a thousand lies: using false photographs to create false childhood memories. Psychonomic Bulletin and Review, 9(3), 597-603. doi:10.3758/BF03196318

Yonelinas, A. P. (2002). The nature of recollection and familiarity: A review of 30 years of research. Journal of Memory and Language, 46(3), 441-517. doi:10.1006/jmla.2002.2864 